Journal for ImmunoTherapy of Cancer

\title{
Expression and clinical significance of PD-L1, B7-H3, B7-H4 and VISTA in craniopharyngioma
}

\author{
Yuelong Wang, ${ }^{1,2}$ Jiaojiao Deng, ${ }^{3}$ Lin Wang, ${ }^{4,5}$ Tingyue Zhou, ${ }^{2}$ Jinlong Yang, ${ }^{1}$ \\ Zerong Tian, ${ }^{1}$ Jinhao Yang, ${ }^{1}$ Hongxu Chen, ${ }^{1}$ Xin Tang, ${ }^{1}$ Shasha Zhao (D) , ${ }^{2}$ \\ Liangxue Zhou, ${ }^{1}$ Aiping Tong, ${ }^{2}$ Jianguo Xu (D) ${ }^{1}$
}

To cite: Wang Y, Deng J, Wang $\mathrm{L}$, et al. Expression and clinical significance of PD-L1, B7-H3, B7-H4 and VISTA in craniopharyngioma. Journal for ImmunoTherapy of Cancer 2020;8:e000406. doi:10.1136/ jitc-2019-000406

- Additional material is published online only. To view, please visit the journal online (http://dx.doi.org/10.1136/jitc2019-000406).

Accepted 02 August 2020

Check for updates

(c) Author(s) (or their employer(s)) 2020. Re-use permitted under CC BY-NC. No commercial re-use. See rights and permissions. Published by BMJ.

${ }^{1}$ Department of Neurosurgery, West China Hospital, Sichuan University, Chengdu, China

${ }^{2}$ State Key Laboratory of Biotherapy and Cancer Center, West China Hospital, Sichuan University, Chengdu, China

${ }^{3}$ Department of Neurosurgery, Huashan Hospital, Fudan University, Shanghai, China

${ }^{4}$ Cardiovascular Institute,

Stanford University School of

Medicine, Stanford, CA, USA

${ }^{5}$ Institute for Stem Cell Biology and Regenerative Medicine,

Stanford University School of

Medicine, Stanford, CA, USA

\section{Correspondence to} Dr Jianguo $\mathrm{Xu}$; jianguo_1229@126.com

Dr Aiping Tong; aipingtong@scu.edu.cn

\section{ABSTRACT}

Background Craniopharyngioma (CP) is a common refractory tumor of the central nervous system. However, little is known about the expression and clinical significance of B7 family ligands/receptors in CP patients. Thus, we conducted the present study to address this issue in a cohort of 132 CP cases.

Methods We mapped and quantified the expression of B7 family ligands/receptors molecules programmed cell death ligand 1 (PD-L1), B7-H3, B7-H4 and V-domain Ig-containing suppressor of $\mathrm{T}$ cell activation (VISTA) in 89 adamantinomatous-type CP and 43 papillarytype CP samples using immunohistochemistry and immunofluorescence. Associations between the marker levels, clinicopathological variables and survival were evaluated.

Results The positive rates of PD-L1, B7-H3, B7-H4 and VISTA in the cohort of $132 \mathrm{CP}$ cases were $76.5 \%$, $100 \%, 40.2 \%$ and $80.3 \%$, respectively. The cut-off values of PD-L1, B7-H3, B7-H4 and PD-L1 expression were determined by survival receiver operating characteristic (ROC) package, which was 70,182, 0 and 20, respectively. Elevated expressions of PD-L1, B7-H3, B7-H4 and VISTA were significantly associated with some clinicopathological characteristics. Kaplan-Meier analysis indicated that higher VISTA expressions correlated with better overall survival (OS) and progression-free survival (PFS) $(p=0.0053$ and $p=0.0066$, respectively). Multivariate Cox regression analysis indicated that VISTA was an independent prognostic factor for OS $(p=0.018)$ but not for PFS $(p=0.898)$.

Conclusions We found variable expression of PD-L1, B7-H3, B7-H4 and VISTA proteins in CPs. The results suggest that the expression level of VISTA may be used as an important indicator to predict the OS and PFS of CPs. B7 family ligands/receptors could be potential immunotherapeutic targets when treating CPs.

\section{INTRODUCTION}

Craniopharyngiomas (CPs) are intracranial nonglial tumors of the sellar and parasellar regions that are most commonly seen in children. ${ }^{1}$ They account for $1.2 \%-4 \%$ of all brain tumors and $6 \%-9 \%$ of all pediatric brain tumors. ${ }^{2}$ CPs are derived from the epithelium of Rathke's pouch. According to WHO classification, CPs can be divided into two types: adamantinomatous-type CP (ACP) and papillary-type CP (PCP). ${ }^{34}$ ACP and PCP are characterized by distinct genetic alterations, such that recurrent activating mutations in CTNNB1 gene coding for $\beta$-catenin were identified in the majority of ACP, whereas, nearly all PCP had recurrent BRAF V600E mutations. ${ }^{56}$

Although the CP is defined by WHO as a benign tumor, both types of CPs are difficult to treat due to their anatomical proximity to important functional structures, such as optic nerve, pituitary stalk, hypothalamus and internal carotid artery. ${ }^{2}$ Surgical resection is the primary treatment for $\mathrm{CP}^{7}{ }^{7}$ However, achieving gross-total resection (GTR) of either type of CP without any complications remains a challenge, ${ }^{8}$ which underlines the necessity of new therapeutic options.

The B7 family ligands/receptors can selectively bind to $\mathrm{T}$ cell ligand to either stimulate or inhibit $\mathrm{T}$ cell responses. ${ }^{9}{ }^{10}$ Immune checkpoint inhibitors targeting the pathway of B7 family members, such as CTLA-4/B7-1/ B7-2 and PD-1/PD-L1, have been shown to provide clinical benefit and transformed the treatment of several advanced cancers, including melanoma and non-small-cell lung cancer. ${ }^{11-13}$ These findings have inspired us to investigate whether these B7 family of ligands/receptors could also be employed as potential therapeutic targets in the treatment of CPs.

Currently, there are very few studies on the expression and function of B7 family ligands/ receptors in CPs. Previous research on ACP $(n=23)$ and PCP $(n=18)$ resections have illustrated that PD-L1 is highly expressed by all tumor cells with a subtype-specific spatial distribution. ${ }^{14}$ This work supports that CPs might be vulnerable to PD-1/PD-L1 immune checkpoint blockade. Coincidentally, we 
have found that another B7 family member B7-H3 is also highly expressed in a small cohort of ACP $(n=30)$ and PCP $(n=14)$ cases. ${ }^{15}$ However, the expression of other B7 family members in CPs remains unknown and the clinical significance of their expressions in CP has not been studied using a large cohort.

Therefore, in this study, we examined the expression of PD-L1, B7-H3, B7-H4 and VISTA proteins in a large CPs cohort using tissue microarray (TMA). In addition, we have correlated the expression of these molecules with overall survival (OS) and progression-free survival (PFS) in this cohort to elucidate the potential association between B7 family of ligands/receptors expression and patients' survival.

\section{MATERIALS AND METHODS \\ Patients and TMA}

The samples were collected from the Department of Neurosurgery of West China Hospital between January 2011 and September 2019, which had adequate tumor samples for TMAs. Written informed consent was obtained from all patients before enrolment. The clinical follow-up was continuously performed until the death of patients. Hospital records and pathology reports of all patients were retrieved and reviewed. Brain tissues were collected from traumatic brain injury patients who underwent injured brain resection. The TMAs were prepared by Shanghai Outdo Biotech (Shanghai, China).

\section{Immunohistochemistry, digital pathology and scoring system}

Immunohistochemical (IHC) staining was performed on TMAs using the following primary antibodies: PD-L1 (1:200, $4.37 \mu \mathrm{g} / \mathrm{mL}, 13684$, Cell Signaling Technology), B7-H3 $(1: 400,1.49 \mu \mathrm{g} / \mathrm{mL}, 14058 \mathrm{~S}$, Cell Signaling Technology), B7-H4 (1:400, $0.66 \mu \mathrm{g} / \mathrm{mL}, 14572$, Cell Signaling Technology) and VISTA (1:200, $0.05 \mu \mathrm{g} / \mathrm{mL}, 54979$, Cell Signaling Technology). ${ }^{15-18}$ TMA slides were stained using standard immunohistochemistry techniques as previously described. ${ }^{15}$ The IHC staining was scanned using Pannoramic MIDI (3D HISTECH). The histoscore of the membrane and nuclear staining quantification was assessed according to the formula $(3+$ per cent cells $) \times 3+(2+$ per cent cells $) \times 2+(1+$ per cent cells $) \times 1$, and the formula total intensity/total cell number was used to assess the histoscore of pixel quantification. In this case, the normalized score is between 0 and 300 . This method has previously been described. ${ }^{19}$

\section{Fluorescent immunohistochemistry}

Immunofluorescence staining (IF) staining was performed on TMAs using the following primary and secondary anti-

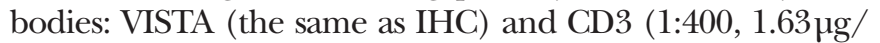
$\mathrm{mL}$, ab699, Abcam), Ionized calcium binding adaptor molecule 1 (IBA-1, 1:100, 25 $\mu \mathrm{g} / \mathrm{mL}$, ab15690, Abcam), PD-L1 (1: 200, $2.5 \mu \mathrm{g} / \mathrm{mL}$, ab210931, Abcam), goat anti-rabbit Alexa594 antibody (1:500, $4 \mu \mathrm{g} / \mathrm{mL}$, ab150088, Abcam), goat anti-

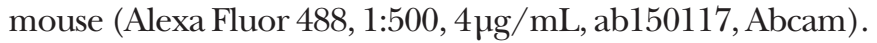

Sections were then cover slipped using hard-set mounting media containing DAPI (ab104139, Abcam).

The fluorescent IHC staining was scanned using Pannoramic MIDI (3D HISTECH). Quantitative measurement of the fluorescence signal was performed using the AQUA method of quantitative IF (QIF) as previously reported. ${ }^{20}$ Briefly, The QIF score of VISTA and PD-L1 signal for each antibody in the tumor were calculated by dividing the target VISTA and PD-L1 pixel intensities in the area of the tumor by the DAPI positivity. Tumor-infiltrating lymphocytes and tumor-associated macrophages (TAMs) were defined as cells that have CD3 and IBA-1 stain positivity, respectively. Scores were normalized to the exposure time and bit depth at which the images were captured, allowing scores collected at different exposure times to be comparable. Cases were considered as target expressers when the QIF score was above the signal detection threshold determined using the negative control preparation and visual inspection.

\section{Polymerase chain reaction and mutation analysis}

Before total DNA from fresh tumor tissue was extracted, the content of the tumor cells was confirmed by instantaneous sections. DNA was extracted by using Animal Genomic DNA Quick Extraction Kit (D0065 M, Beyotime, China). Primer pairs were used for gene amplification of CTNNB1 exon 3 (Primer sequence Forward: 5'-TCACTGAGCTAACCCTGGCT-3', Reverse: 5'- GCATTCTGACTTTCAGTAAG GCAAT-3') and BRAF V600E (Primer sequence Forward: 5'AGGAAAGCATCTCACCTCATCCT-3', Reverse: 5'-AGGA AAGCATCTCACCTCATCCT-3') . PCR was performed by applying the default conditions: $5 \mathrm{~min} 95^{\circ} \mathrm{C}$ for initial denaturation, which was followed by 30 cycles of amplification that consist of denaturation at $95^{\circ} \mathrm{C}$ for $60 \mathrm{~s}$, annealing at $60^{\circ} \mathrm{C}$ for $45 \mathrm{~s}$, and extension at $72^{\circ} \mathrm{C}$ for $90 \mathrm{~s}$ with final extension $72^{\circ} \mathrm{C}$ for $12 \mathrm{~min}$. PCR products were sent to TsingKe for sequencing analysis.

\section{Statistical analysis}

All values are expressed as means \pm SEM. Statistical data analysis was performed using GraphPad Prism V.5.03 (GraphPad Software La Jolla, California, USA) statistical package and R language (V.3.6.1). Interdependence between staining and clinical data was calculated using the $\mathrm{X}^{2}$ displayed by crosstables. Student's t-test was used for two-group analysis. The Cox proportional hazards regression model and KaplanMeier plots were generated by the survival package, and the log-rank test was used to compare survival curves between high and low expression groups. The cut-off points were determined by the survivalROC package. Statistical significance was defined as a $\mathrm{p}<0.05$.

\section{RESULTS}

\section{Patient clinicopathological characteristics and outcome}

A total of 132 patients were enrolled, including 86 males and 46 females (male/female ratio 1.86:1, table 1, online supplemental table 1). Patients' ages had a bimodal distribution and ranged from 2 to 75 years with the median age of 40 . We chose the lowest point (35 years) between 


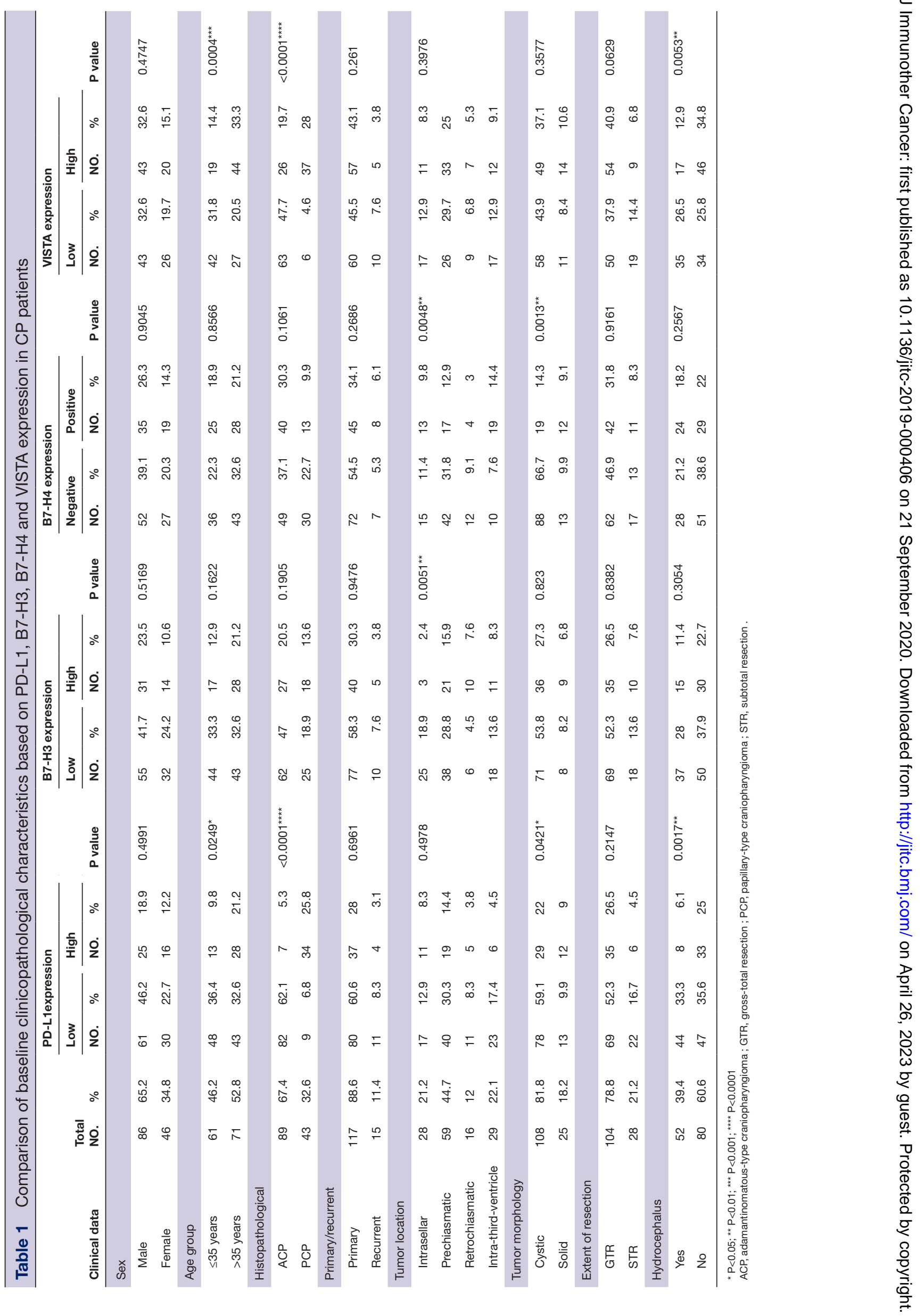


the two peaks to stratify age. Pathologically, there were 89 ACPs and 43 PCPs. Within this cohort, 117 patients had primary tumors and 15 patients had recurrent tumors. The clinical presentations of patients in the study included the following: vision loss, headache, fatigue, drowsiness and unconsciousness. The average tumor diameter in this cohort was $3.6 \mathrm{~cm}$ (ranges from 1.2 to $9.2 \mathrm{~cm}$ ). GTR and subtotal resection (STR) were achieved in $104(78.8 \%)$ and $28(21.2 \%)$ cases, respectively.

A total of 110 out of 132 patients $(83.3 \%)$ were followed up for an average of 18.2 months, which ranges from 0.2 to 84 months (online supplemental table 1 ). Within the perioperative period, 26 deaths have occurred. For the 87 GTR patients being followed up, 6 (6.9\%) had shown tumor recurrence within an average of 30 months, which ranges from 2 to 82 months. Among the 28 STR patients being followed up, $10(35.7 \%)$ had experienced tumor recurrence within an average of 16.2 months, which ranges from 6 to 53 months.

In this cohort, the cut-off values of PD-L1, B7-H3, B7-H4 and PD-L1 expressions were determined by survivalROC package, which was 70, 182, 0 and 20, respectively. Elevated expressions of PD-L1, B7-H3, B7-H4 and VISTA were significantly associated with some clinicopathological characteristics (table 1). For example, PD-L1 expression was associated with age, histopathological, tumor morphology and hydrocephalus, whereas VISTA expression was connected with age, histopathological and hydrocephalus.

\section{Expression of B7 family proteins in CP tumor cells}

Using IHC, we explored the expression characteristics of PD-L1, B7-H3, B7-H4 and VISTA in CP. Consistent with the previous study, ${ }^{12}$ the patterns of PD-L1 staining were predominantly membranous, and $101(76.5 \%)$ positive cases were detected in this set of data. In ACP, PD-L1 expression was present in the regions of well-keratinized squamous tumor epithelium (figure 1A). Notably, we also found strong staining around the cholesterol crystals, which was not seen in previous reports (figure 1A). Compared with ACP, PCP had a much higher expression of PD-L1 (figure 1B), which was observed in multiple layers of tumor cells with the strongest expression surrounding the fibrovascular stroma and decreased with increasing distance from the stroma (figure 1A).

B7-H3 was detectable to varying degrees in all samples $(100 \%)$. It was mainly expressed on the cell membrane of tumor cells and was also observed in vascular endothelial cells and tumor-infiltrating immune cells in the two subtypes of CPs (figure 1A). The average expression scores of $\mathrm{B} 7-\mathrm{H} 3$ in both ACP and PCP were higher than in normal brain tissues, but no significant difference was detected between ACP and PCP (figure 1B). Intriguingly, we also found that less than one half of $\mathrm{CP}$ tissues $(53 / 132,40.2 \%)$ have weak to moderate expression of $\mathrm{B} 7-\mathrm{H} 4$ (figure 1A), which is currently reported to be expressed only in a few type of tumor cells. ${ }^{21} \mathrm{~B} 7-\mathrm{H} 4$ mainly expressed on the surface of CP cell membrane far from the basal cells. There was no difference in expression level between the two subtypes of CPs (figure 1B).

IHC staining demonstrated that $106(80.3 \%)$ CP specimens, including 67 ACPs (histoscore range from 10 to 90 ) and 39 PCPs (histoscore range from 10 to 230), were positive for VISTA staining. The expression of VISTA was detected both on the cell membrane and in the cytoplasm (figure 1A). It was predominantly presented in the regions of epithelial whorls in ACP and on multiple layers of tumor cells which are far from the basal cell in PCP (figure 1A). The VISTA histoscore was significantly higher in PCPs than in brain tissues and ACPs. However, we found no statistical significance between normal brain tissues and ACPs (figure 1B).

We further compared the expression of PD-L1, B7-H3, B7-H4 and VISTA in primary tumors versus recurrent tumors as well as between preoperative radiotherapy and non-radiotherapy groups. The results suggested that there was no significant difference between the groups (online supplemental figure 1).

\section{Expression of B7 family proteins in tumor-associated immune cells}

All CP cases contain numerous immune cells in the stroma and epithelium. Among these cases, we also found that tumor-associated immune cells expressed B7 family ligands/receptors, of which VISTA expression was the highest (figure 2A). To explore the possible role of VISTA in the immune microenvironment of CPs, we measured its level in $\mathrm{CD}^{+}$tumor-infiltrating $\mathrm{T}$ cells and IBA- $1^{+}$TAMs by colocalization IF staining. As shown in figure 2B-D, VISTA was detected in all immune cell subsets but was significantly higher in IBA- $1^{+}$TAMs than in $\mathrm{CD} 3$ positive lymphocyte in both ACP (figure 2C) and PCP (figure 2D). Additionally, we found that there was a negative association between CD3 and VISTA, and a positive association between VISTA and IBA-1 (figure 2E-F).

\section{B7 family proteins expression and CTNNB1 and BRAF mutation status}

We further analyzed CTNNB1 and BRAF mutation status in CPs by PCR. 60\% (21 out of 35) cases exhibited activating mutations within exon 3 of the $\beta$-catenin gene CTNNB1 in ACPs (figure 3A) and 56\% (14 out of 25) cases exhibited BRAF V600E mutation in PCPs (figure 3B). In ACPs, CTNNB1 mutation could be linked to the high expression of PD-L1, B7-H3 and VISTA. Moreover, there was a significant difference $(\mathrm{p}=0.0363)$ in $\mathrm{B} 7-\mathrm{H} 3$ expression between the two subtypes of CP (figure 3A). Notably, in PCPs, BRAF V600E mutation group showed a significantly higher VISTA expression comparing to the wild type group $(\mathrm{p}=0.0117)$ (figure 3B).

\section{Clinical significance of B7 family proteins expression in CPs}

We further analyzed the relationship between CP tumor size and B7 family ligands/receptors expression. We found that VISTA expression and tumor size had an inverse association $\left(\mathrm{R}^{2}=0.04871, \mathrm{p}=0.0110\right)$; but the expression 

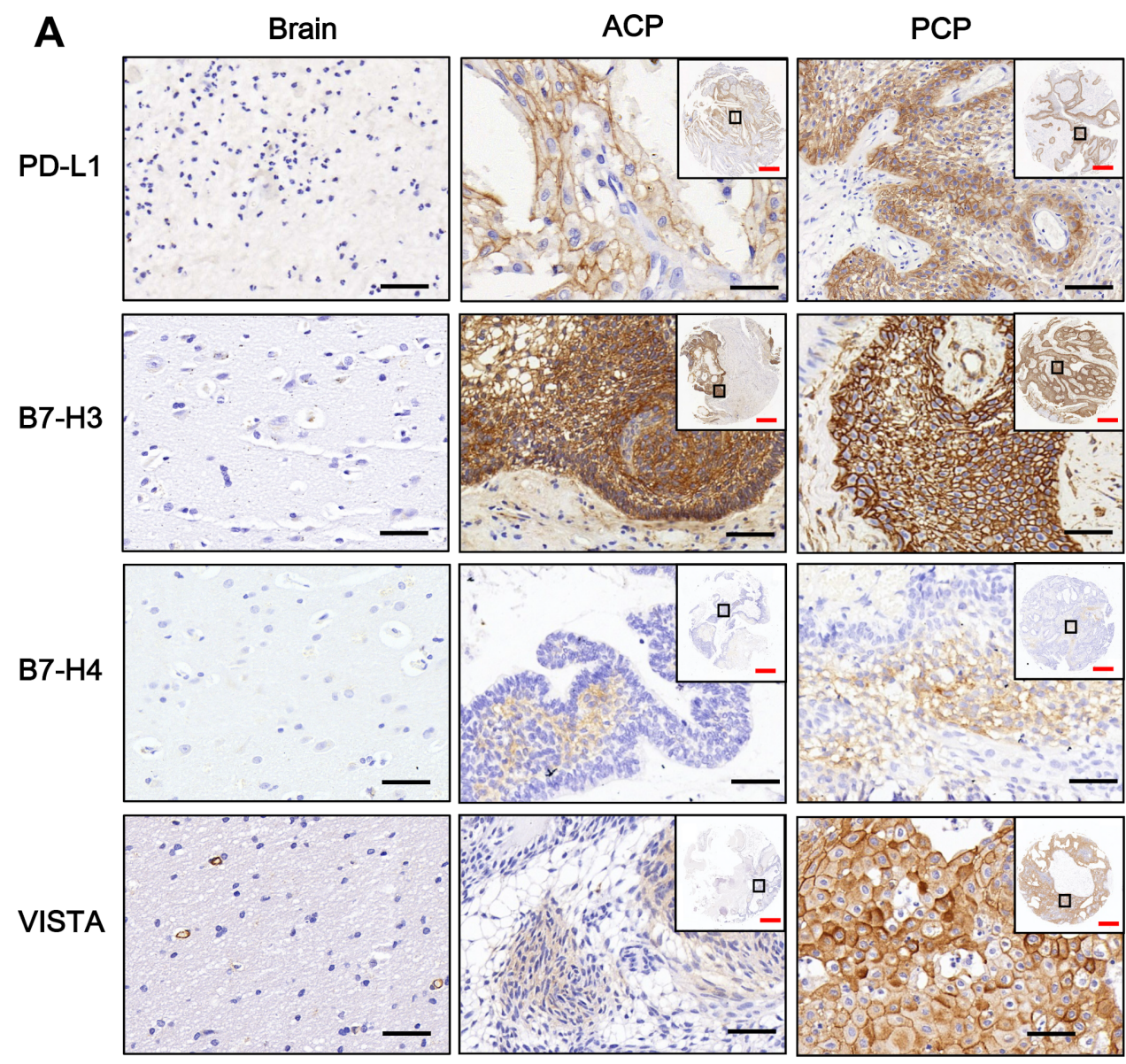

B
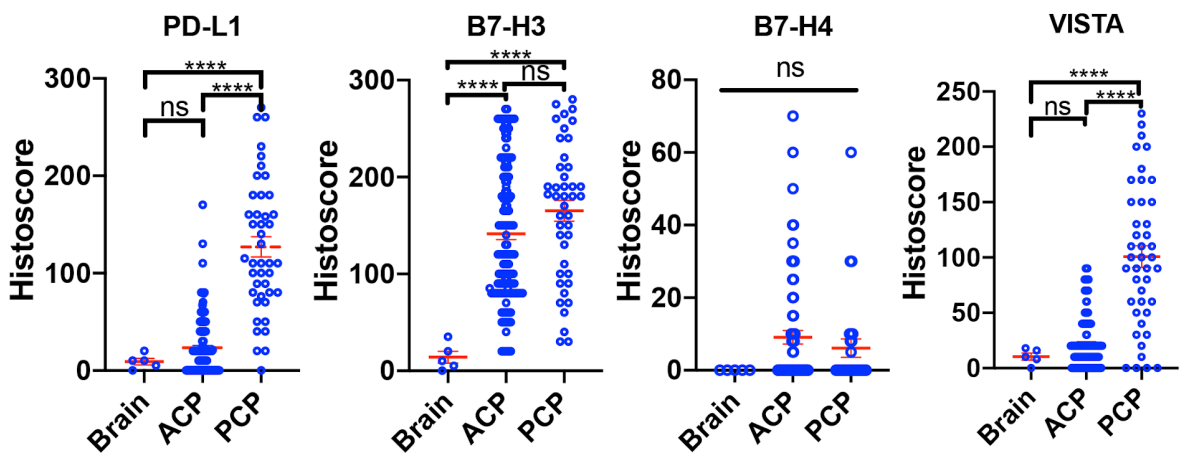

Figure 1 Expression of the B7 family ligands/receptors on CPs. (A) PD-L1, B7-H3, B7-H4 and VISTA expression on the brain, ACP and PCP. (B) Comparison of biomarker expression status in different tumor subtypes. All data displays mean \pm SEM.

Red bar: $0.5 \mathrm{~mm}$, black bar: $50 \mu \mathrm{m} .{ }^{* \star \star *} \mathrm{P}<0.0001$. ACP, adamantinomatous-type craniopharyngioma; ns: no significant; PCP, papillary-type CP.

of the other three molecules had no association with tumor size (figure 4A). Kaplan-Meier survival curves by B7 family ligands/receptors expression levels are shown in figure 4B. Interestingly, strong VISTA expression was correlated with favorable outcomes in CPs. Calculated median OS in CP patients with high VISTA expression was 7 years compared with a median OS of 2.25 years in VISTA negative or low patients $(\mathrm{p}=0.0027)$. However, elevated expression of PD-L1, B7-H3 or B7-H4 was not associated with OS nor PFS in this cohort.

The univariate Cox regression model revealed that histopathological, tumor location, the extent of resection and VISTA expression were all correlated with PFS $(\mathrm{p}<0.05)$, whereas tumor location, hydrocephalus and VISTA expression were associated with OS (table 2). We selected the characteristics with $\mathrm{p}<0.1$ in univariate for multivariate analysis. After adjustment, we found that only VISTA was an independent prognostic factor for OS $(p=0.018)$, while only the extent of resection was an independent prognostic factor for PFS of CP patients $(\mathrm{p}=0.025)$.

\section{Increased expression of VISTA correlated with PD-L1}

Recently, some reports showed that VISTA overexpression is correlated with other B7 family ligands/receptors, such as PD-L1, PD-1 and CTLA-4. When investigating the 

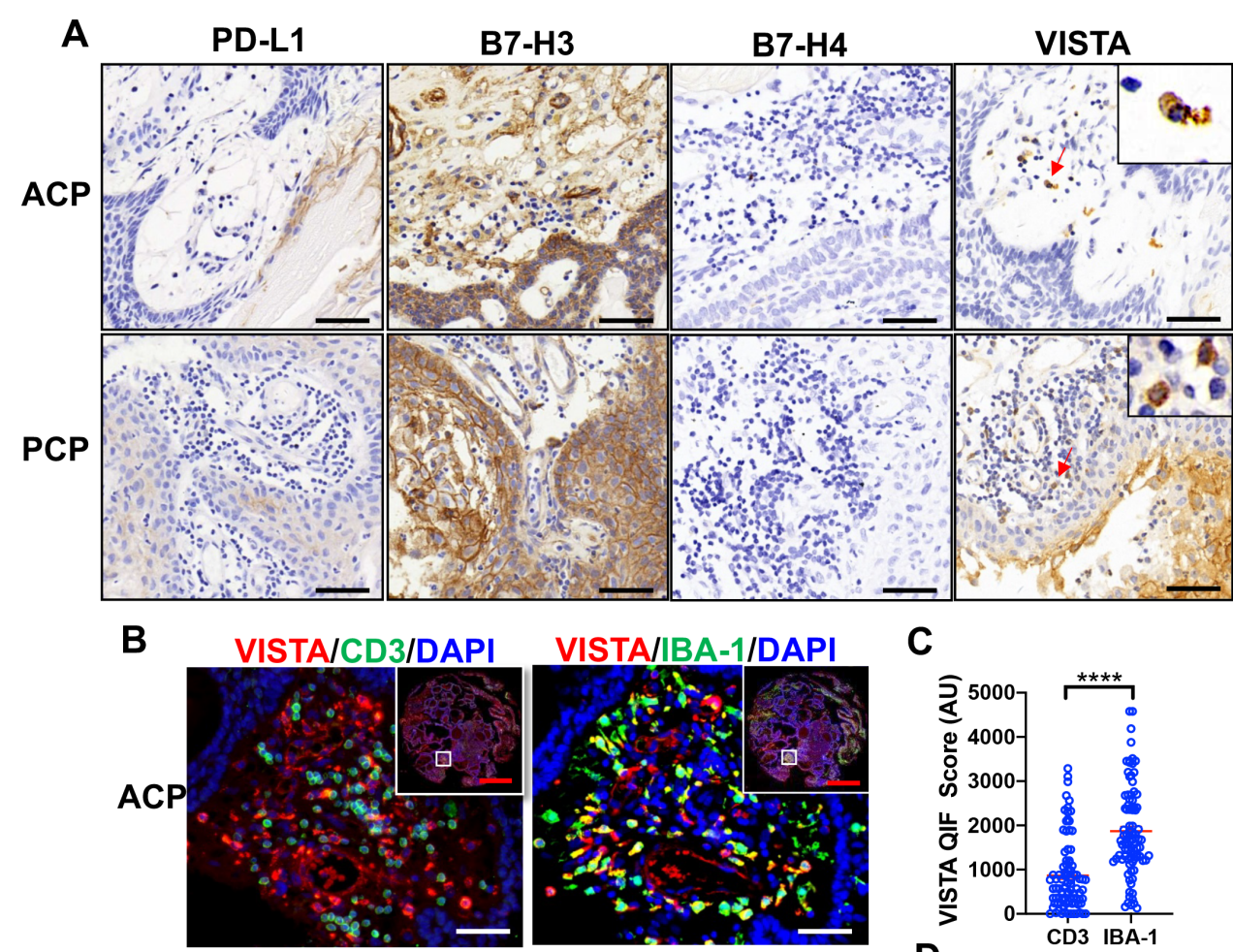

C
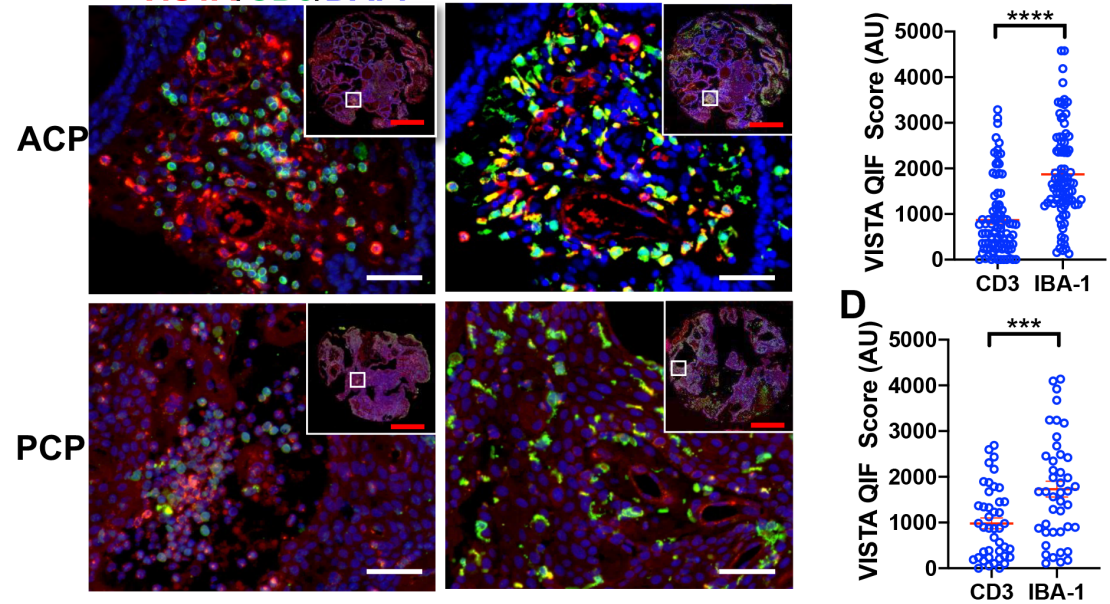

E
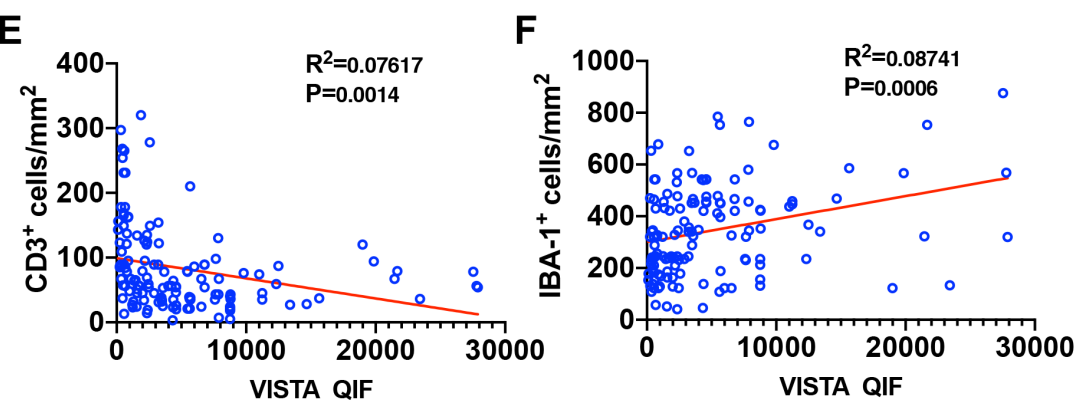

Figure 2 Expression of the B7 family ligands/receptors on immune cells of CPs. (A) PD-L1, B7-H3, B7-H4 and VISTA expression on immune cells in ACP and PCP. The red arrow refers to the immune cells with positive VISTA. (B) Representative images of VISTA, CD3 and IBA-1 codetection are shown. (C, D) QIF scores show IBA- $1^{+}$tumor-associated macrophage expressed more VISTA than $\mathrm{CD}^{+}$T lymphocytes in both ACP and PCP. VISTA expression is negatively correlated with the number of $\mathrm{CD}^{+}$cells $(\mathrm{E})$ in the tumor and positively correlated with the number of IBA-1 ${ }^{+}$cells $(\mathrm{F})$. Red bar: $0.5 \mathrm{~mm}$, black bar: $50 \mu \mathrm{m}$, white bar: $50 \mu \mathrm{m} .{ }^{* \star *} \mathrm{P}<0.001,{ }^{* \star \star *} \mathrm{P}<0.0001$. ACP, adamantinomatous-type craniopharyngioma; PCP, papillary-type CP; QIF, quantitative immunofluorescence.

molecules potentially associated with VISTA in the tumor microenvironment, we observed that VISTA expression was associated with PD-L1 in CPs. However, there was no significant association between the expression of VISTA and the expression of $\mathrm{B} 7-\mathrm{H} 3$ and $\mathrm{B} 7-\mathrm{H} 4$ (figure 5A). Furthermore, we explored the spatial distribution relationship between the expression of PD-L1 and VISTA in tumor tissues by colocalization IF staining. We found that PD-L1 and VISTA colocalized with each other only in immune cells within the tumor stroma but not in tumor cells (figure 5B,C).

\section{DISCUSSION}

Immunotherapy holds great promise for eradicating cancer and has achieved long term benefits as well as improved clinical outcomes in treating various types of advanced malignancies, such as late-stage metastatic 


\section{A}
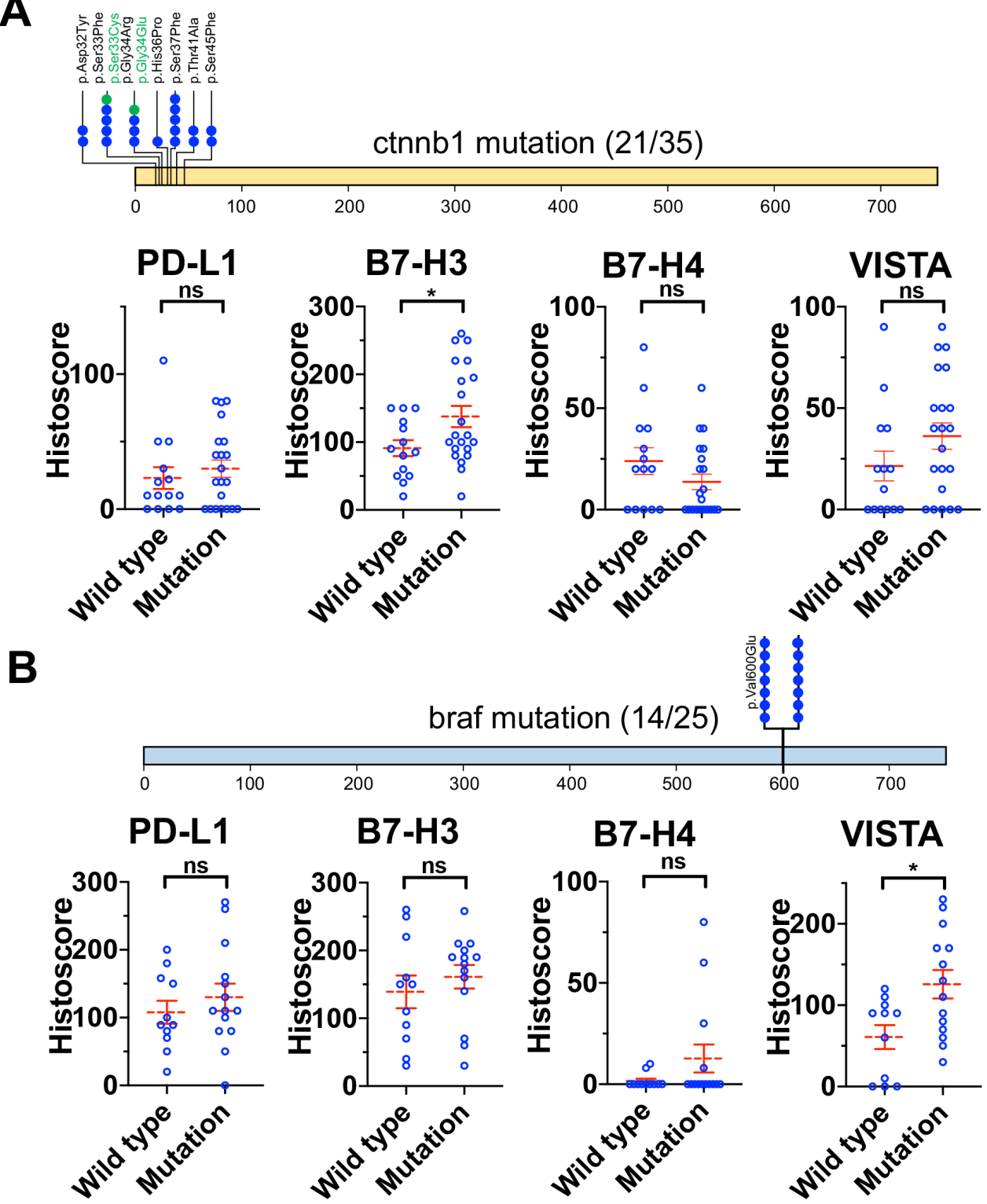

Figure 3 Gene mutation and its relationship with B7 family ligands/receptors expression. CTNNB1 mutation was detected in 21 out of 35 ACPs (A). The expression of B7-H3 in the mutation group was significantly higher than in the wild type group, but there was no statistical difference between the two groups. BRAF mutation was detected in 14 out of 25 PCPs (B). The expression of VISTA was higher in the mutation group than in the wild type group, and this result is statistically significant. ${ }^{*} \mathrm{P}<0.05$. ACP, adamantinomatous-type craniopharyngioma; ns, no significant; PCPs, papillary-type CP.

melanoma and non-small cell lung cancer. ${ }^{22}$ Immune checkpoint receptors through binding to their ligands can deliver coinhibitory signals to negatively regulate $T$ cell responses, which will facilitate tumor escape from immune surveillance. Thus, many immune checkpoint receptors have been identified as potential cancer therapeutic targets, and among them, B7 family ligands/receptors have received increasing attention. ${ }^{23}$ The expanding list of B7 family members includes CTLA-4 (ligands B7.1 and B7.2), PD-1 (ligands PD-L1 and PD-L2), TIM3 (ligands CEACAM-1, HMGB1 and galectin-9), TIGIT (ligands CD155 and CD112), BTLA (ligand HVEM), B7-H3, B7-H4 and VISTA. ${ }^{24}$ In this study, we investigated the expression level of B7 family ligands/receptors in CPs. More importantly, we have discovered some associations between B7 family ligands/receptors expression and clinical features of CPs.

Using IHC, we have shown that PD-L1, B7-H3, B7-H4 and VISTA are differentially expressed both in ACPs and PCPs. These B7 family ligands/receptors are preferentially located on the membrane of tumor cells. Previously, some researches demonstrated that radiation could upregulate B7 family ligands/receptors expression. ${ }^{26}{ }^{27}$ However, we found that there was no significant difference in the expression of PD-L1, B7-H3, B7-H4 and VISTA between preoperative radiotherapy and non-radiotherapy groups. A few factors may contribute to the different results that we obtained. First, we had a relatively small sample size, and only five relapsed patients and one primary patient received radiotherapy before surgery. Among them, 

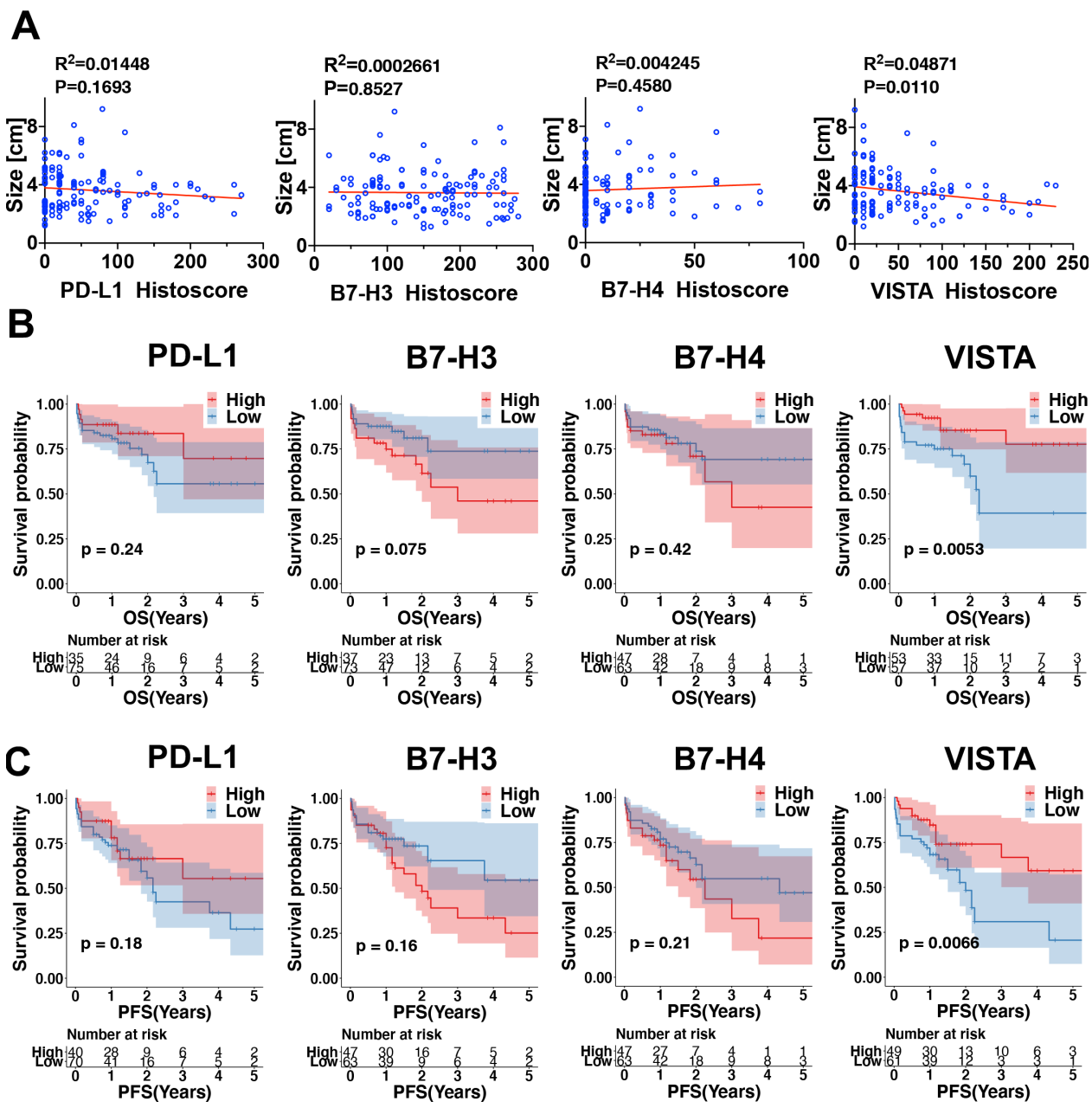

Figure 4 Prognostic value of the B7 family ligands/receptors in CPs. Analysis of the relationship between the expression of B7 family ligands/receptors and tumor volume (A). Kaplan-Meier curve for overall survival (B) and progression-free survival (C) based on B7 family ligands/receptors expression. CPs, craniopharyngioma; OS, overall survival; PD-L1, programmed cell death ligand 1; PFS, progression-free survival.

3 patients received general radiotherapy, and the rest received gamma knife therapy. Second, there was a long duration between patients who received radiotherapy and underwent reoperation. The effects of radiation on the tumor might be weakened.

Interestingly, we also observed that VISTA is highly expressed on tumor-associated immune cells. Moreover, by IF colocalization staining, we found that VISTA has a higher expression on macrophages than $\mathrm{T}$ lymphocytes. Former studies have revealed the high expression of VISTA in hematopoietic cells, lung and small intestine in mice. ${ }^{28}$ Additionally, VISTA has also been reported to be mainly expressed in hematopoietic cells, especially in myeloid cells in human. ${ }^{2029}{ }^{30}$ However, only a few articles reported that VISTA is expressed on tumor cells. ${ }^{2031} 32$ To our knowledge, we are the first group to demonstrate that VISTA has a high expression on tumor cells in a large human CPs cohort.

It has been reported that the expression of $\mathrm{B} 7$ family ligands/receptors regulators in various tumors is associated with different prognoses. For example, a recent study revealed that the OS and disease-free survival outcomes in patients with hepatocellular carcinoma negatively correlate with the expression level of PD-L2 on tumor membranes and PD-L1 in the immune stroma. Besides, Altan $e t a \hat{l}^{33}$ have described that the high $\mathrm{B} 7-\mathrm{H} 3$ protein level (top 10 percentile) was associated with poor OS in NSCLC. Loeser $e t a p^{34}$ have also found that esophageal adenocarcinoma patients with VISTA expression have demonstrated an improved median OS compared with the patients without VISTA expression (202.2 months vs 21.6 months; $\mathrm{p}<0.0001)$. In our study, we identified that patients with high expression of VISTA had longer OS and FPS, while the expression of PD-L1, B7-H3 and B7-H4 was not related to prognosis in CPs. Thus, VISTA may serve as an important prognostic marker for CPs. However, other validation cohorts need to be used to demonstrate the consistency of the findings and validity of the marker cut-off values in future research.

In this work, we also analyzed the relationship between the expression of B7 family ligands/receptors and clinical significance. VISTA expression was statistically correlated with patient age $(\mathrm{p}=0.0004)$, tumor histopathological subtypes $(\mathrm{p}<0.0001)$ and hydrocephalus $(\mathrm{p}=0.0053)$. CPs are the most common pediatric intracranial tumors of nonglial origin. ${ }^{12}$ These tumors can present at any age, 


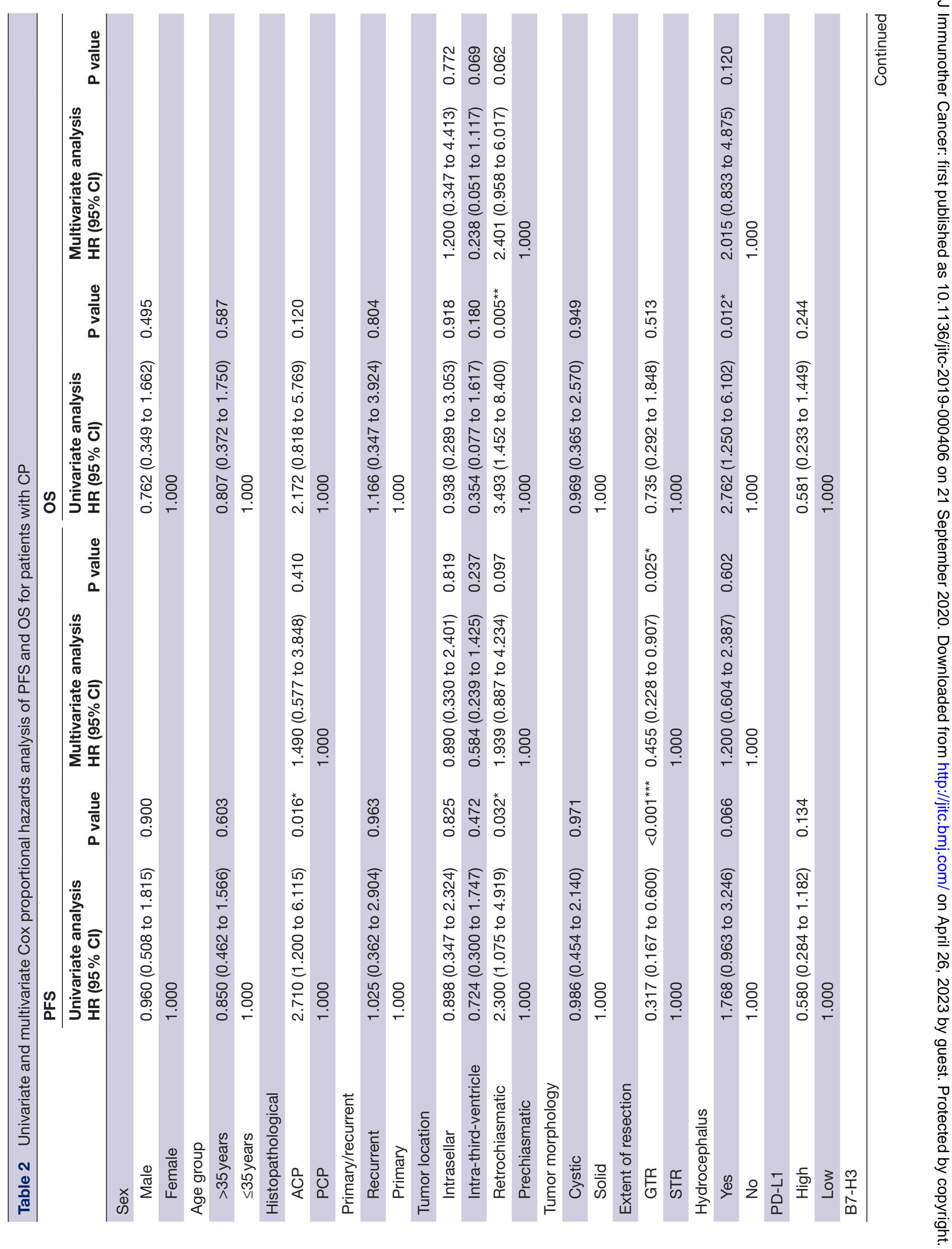




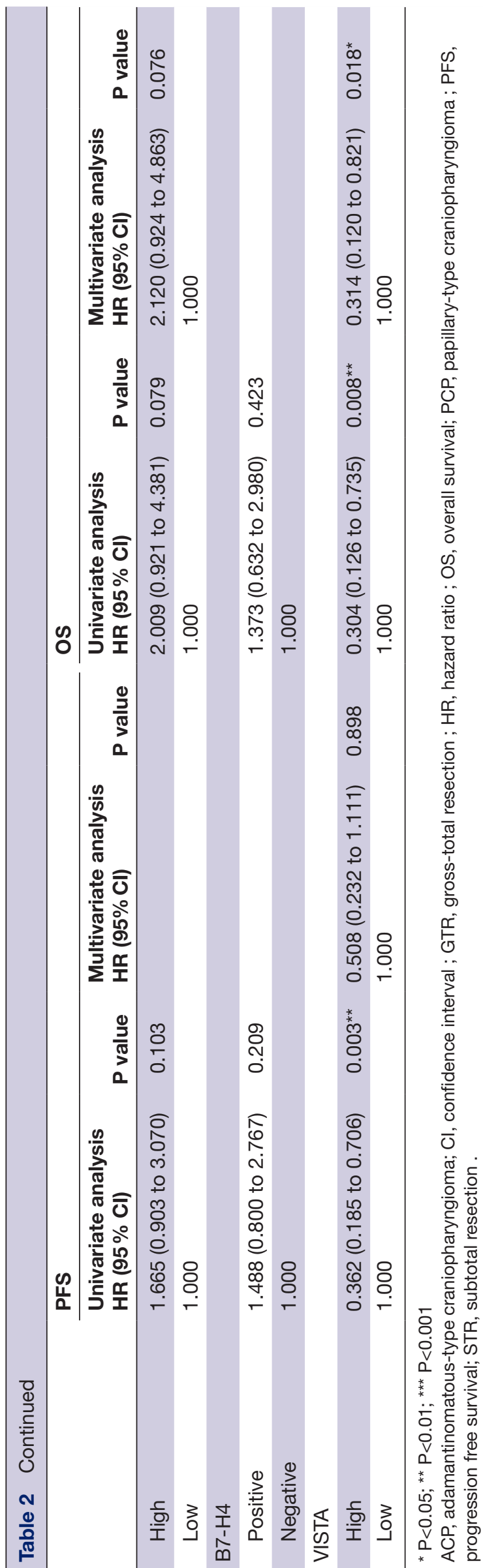

there is a bimodal age distribution, with peak incidence rates in children aged $5-15$ years and in adults aged $40-70$ years. Among them, the PCP subtype is mainly found in adults but very rare in children. In our study, we found that PCP has higher VISTA expression than ACP (figure 1 and table 1). This may explain why the high expression of VISTA is mainly distributed in adults.

Most PCP patients carry recurrent activating mutations in BRAF V600E. ${ }^{35} 36$ This mutation causes constitutive activation of the BRAF kinase and subsequent oncogenic activation of the mitogen-activated protein kinase pathway through phosphorylating MEK and ERK. ${ }^{37}$ Therefore, we hypothesized that PCPs with BRAF V600E mutation has higher VISTA expression. By comparing the expression of VISTA between the mutant group and the wild type group, we found that the BRAF mutant group indeed had a higher expression of VISTA. We suspect that the RAS/RAF/MEK/ERK signaling pathway may play an important role in regulating VISTA expression in PCPs.

Currently, little is known about the association between VISTA and other B7 family members. In our work, we observed that VISTA was positively correlated with PD-L1. Moreover, we found that the expression and distribution of VISTA and PD-L1 on the surface of tumor cells were significantly different. PD-L1 was highly expressed in the basaloid, while the high expression of VISTA was seen far away from the basaloid (figure 5B). This finding suggests that VISTA and PD-L1 are regulated by different mechanisms in tumor cells. It is possible that the tumor cells far away from the basaloid are also far away from the tumor blood vessels, thus, the tumor microenvironment is relatively anoxic. Therefore, we speculate that hypoxia may regulate the expression of VISTA in CP cells. A study using a CT26 colon cancer model showed that compared with less hypoxic tumor regions or draining lymph nodes, regions of profound hypoxia in the tumor microenvironment were associated with increased VISTA expression on tumor-infiltrating myeloid-derived suppressor cells. ${ }^{38}$ Using chromatin immunoprecipitation and genetic silencing, this study showed that hypoxia-inducible factor- $1 \alpha$ binds to a conserved hypoxia response element in the VISTA promoter region and upregulates VISTA on myeloid cells. ${ }^{38}$

Notably, our study also has several limitations. First, we included retrospectively collected samples from cases with variable follow-ups as well as different treatments, and the selection bias cannot be excluded. Second, the use of TMAs to examine a relatively small tissue area could over-represent or under-represent the markers in the cases. Third, in this study, patients who died in the short term after operation were not excluded. The deaths in this scenario are mainly due to serious surgical complications caused by surgical resection of CP. The inclusion of these cases may interfere with the evaluation of prognosis. Finally, further experiments are needed to verify the role of $\mathrm{B} 7$ family ligands/receptors in the development of CP. 
A

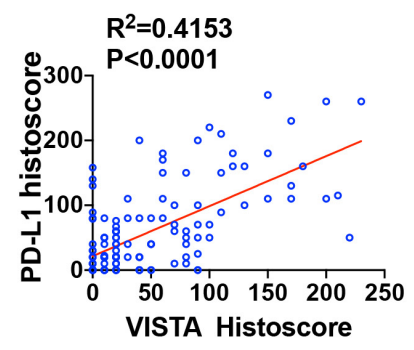

B

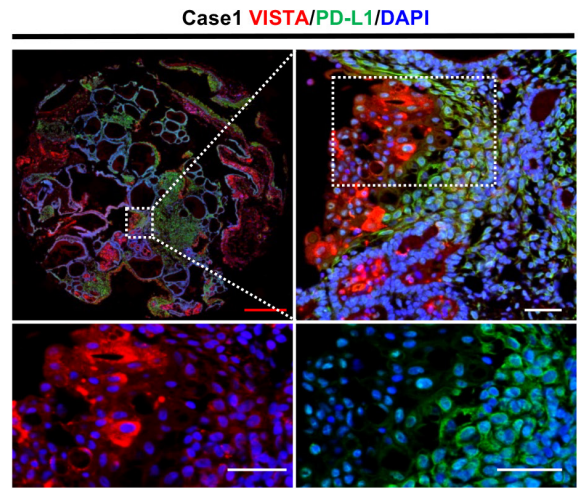

C

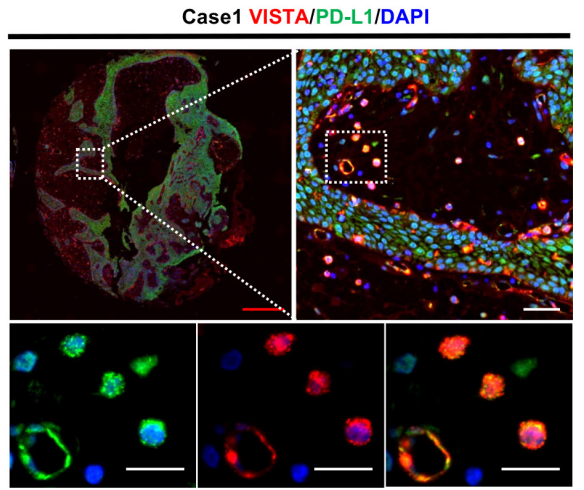

$R^{2}=0.02895$

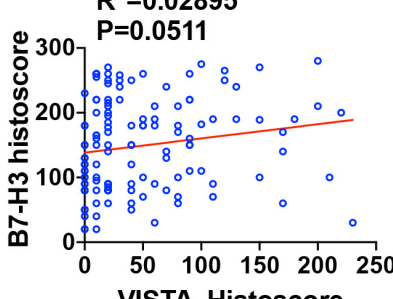

VISTA Histoscore

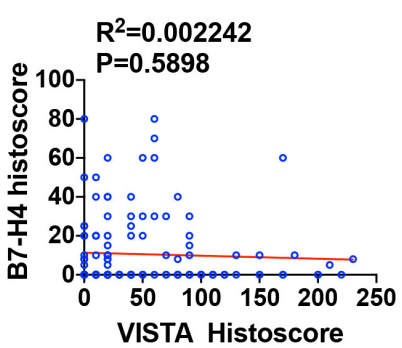

Case2 VISTA/PD-L1/DAPI

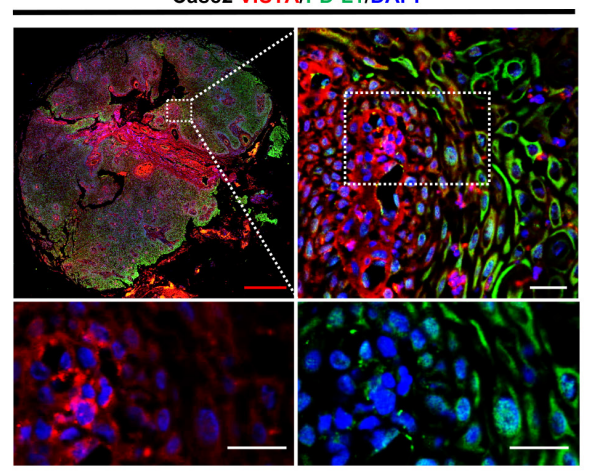

Case2 VISTA/PD-L1/DAPI

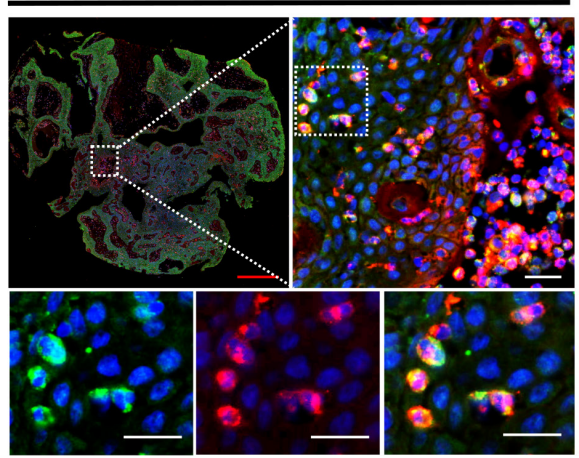

Figure 5 VISTA is positively correlated with PD-L1 in CPs. Analysis of the correlation between the expression of VISTA and the expression of PD-L1, B7-H3 and B7-H4 in CPs (A). Immunofluorescence colocalization revealed the difference that expressions of PD-L1 and VISTA were different in tumor cells (B), but the two molecules were colocalized in tumor associated immune cells (C). Red bar: $0.5 \mathrm{~mm}$, white bar: $50 \mu \mathrm{m}$. CPs, craniopharyngioma; PD-L1, programmed cell death ligand 1 .

\section{CONCLUSION}

We performed a comprehensive evaluation of B7 family molecules in CPs and found heterogeneous expression of PD-L1 in CPs. B7-H3, and VISTA proteins were highly expressed in CPs, while B7-H4 was expressed at a lower level. Our study also revealed that the expression level of VISTA may be utilized as an important indicator to predict the OS and PFS of CPs. The wide expression of PD-L1, B7-H3 and VISTA in human CPs, together with their immunomodulatory capacity, all suggests that these B7 family ligands/receptors may serve as both prognostic markers and attractive novel targets for immunotherapy against CPs.

Contributors Study conception and design: YW, AT and JX. Staining of clinical cases and image analysis: YW, JD, ZT, JingY and JinhY. Statistical analyses: YW, AT, TZ and SZ. Drafting of manuscript: YW, LW and AT. Critical revision of manuscript: all authors. Supervision and securing of study resources: LZ and AT. All authors read and approved the final manuscript.

Funding This work was supported by 1.3.5 project for disciplines of excellence, West China Hospital, Sichuan University(ZYJC18007); Key research and development project of science and technology department of Sichuan Province(2019YFS0326, 2019YFS0392); Postdoctoral Research Fund of China
(2018M640922); National Natural Science Foundation of China (81772693); Postdoctoral Research Fund of West China Hospital (19HXBH055).

Competing interests None declared.

Patient consent for publication Not required.

Ethics approval This study was approved by the Medical Ethics Committee of Hospital of West China Hospital of Sichuan University Biomedical Ethics Committee and was performed according to the Institutional Guidelines.

Provenance and peer review Not commissioned; externally peer reviewed.

Data availability statement All data relevant to the study are included in the article or uploaded as online supplementary information. Deidentified datasets used and/or analyzed during the current study are available from the corresponding author on reasonable request.

Open access This is an open access article distributed in accordance with the Creative Commons Attribution Non Commercial (CC BY-NC 4.0) license, which permits others to distribute, remix, adapt, build upon this work noncommercially, and license their derivative works on different terms, provided the original work is properly cited, appropriate credit is given, any changes made indicated, and the use is non-commercial. See http://creativecommons.org/ licenses/by-nc/4.0/.

\section{ORCID iDs}

Shasha Zhao http://orcid.org/0000-0003-4775-614X

Jianguo Xu http://orcid.org/0000-0001-9651-8186 


\section{REFERENCES}

1 Müller HL. Craniopharyngioma. Endocr Rev 2014;35:513-43.

2 Müller HL, Merchant TE, Puget S, et al. New outlook on the diagnosis, treatment and follow-up of childhood-onset craniopharyngioma. Nat Rev Endocrinol 2017;13:299-312.

3 Cala LA, Robbins PD. The impact of the 2016 World Health organization classification of tumours of the central nervous system upon diagnosis and prognosis. Australasian Med J 2017;10:426-31.

4 Wang Y, Deng J, Guo G, et al. Clinical and prognostic role of annexin A2 in Adamantinomatous craniopharyngioma. J Neurooncol 2017;131:21-9.

5 Brastianos PK, Taylor-Weiner A, Manley PE, et al. Exome sequencing identifies BRAF mutations in papillary craniopharyngiomas. Nat Genet 2014;46:161-5.

6 Malgulwar PB, Nambirajan A, Pathak P, et al. Study of $\beta$-catenin and BRAF alterations in Adamantinomatous and papillary craniopharyngiomas: mutation analysis with immunohistochemical correlation in 54 cases. J Neurooncol 2017;133:487-95.

7 Robinson LC, Santagata S, Hankinson TC. Potential evolution of neurosurgical treatment paradigms for craniopharyngioma based on genomic and transcriptomic characteristics. Neurosurg Focus 2016:41:E3.

8 Shi Xiang'en, Zhou Z, Wu B, et al. Outcome of radical surgical resection for craniopharyngioma with hypothalamic preservation: a single-center retrospective study of 1054 patients. World Neurosurg 2017;102:167-80.

9 Ni L, Dong C. New B7 family checkpoints in human cancers. Mol Cancer Ther 2017;16:1203-11.

10 Carvajal-Hausdorf D, Altan M, Velcheti V, et al. Expression and clinical significance of PD-L1, B7-H3, B7-H4 and TILs in human small cell lung cancer (SCLC). J Immunother Cancer 2019;7:1-9.

11 Sun C, Mezzadra R, Schumacher TN. Regulation and function of the PD-L1 checkpoint. Immunity 2018;48:434-52.

12 Mok TSK, Wu Y-L, Kudaba I, et al. Pembrolizumab versus chemotherapy for previously untreated, PD-L1-expressing, locally advanced or metastatic non-small-cell lung cancer (KEYNOTE-042): a randomised, open-label, controlled, phase 3 trial. Lancet 2019;393:1819-30.

13 Rodig SJ, Gusenleitner D, Jackson DG, et al. MHC proteins confer differential sensitivity to CTLA-4 and PD-1 blockade in untreated metastatic melanoma. Sci Trans/ Med 2018;10:eaar3342.

14 Coy S, Rashid R, Lin J-R, et al. Multiplexed immunofluorescence reveals potential PD-1/PD-L1 pathway vulnerabilities in craniopharyngioma. Neuro Oncol 2018;20:1101-12.

15 Chen C, Wang Y, Zhong K, et al. Frequent B7-H3 overexpression in craniopharyngioma. Biochem Biophys Res Commun 2019;514:379-85.

16 Qin G, Wang X, Ye S, et al. NPM1 upregulates the transcription of PD-L1 and suppresses T cell activity in triple-negative breast cancer. Nat Commun 2020;11:1-16.

17 Liang L, Jiang Y, Chen J-S, et al. B7-H4 expression in ovarian serous carcinoma: a study of 306 cases. Hum Pathol 2016;57:1-6.

18 Deng J, Ma M, Wang D, et al. Expression and clinical significance of immune checkpoint regulator B7-H3 (CD276) in human meningioma. World Neurosurg 2020;135:e12-18.

19 Sun Z-J, Zhang L, Hall B, et al. Chemopreventive and chemotherapeutic actions of mTOR inhibitor in genetically defined head and neck squamous cell carcinoma mouse model. Clin Cancer Res 2012;18:5304-13.

20 Villarroel-Espindola F, Yu X, Datar I, et al. Spatially resolved and quantitative analysis of VISTA/PD-1H as a novel immunotherapy target in human non-small cell lung cancer. Clin Cancer Res 2018;24:1562-73.

21 Schalper KA, Carvajal-Hausdorf D, McLaughlin J, et al. Differential expression and significance of PD-L1, IDO-1, and B7-H4 in human lung cancer. Clin Cancer Res 2017;23:370-8.

22 McNutt M. Cancer immunotherapy. Science 2013;342:1417.

23 Byun DJ, Wolchok JD, Rosenberg LM, et al. Cancer immunotherapy - immune checkpoint blockade and associated endocrinopathies. Nat Rev Endocrinol 2017;13:195-207.

24 Horn LA, Riskin J, Hempel HA, et al. Simultaneous inhibition of CXCR1/2, TGF- $\beta$, and PD-L1 remodels the tumor and its microenvironment to drive antitumor immunity. J Immunother Cancer 2020;8:e000326.

25 Sharma P, Allison JP. The future of immune checkpoint therapy. Science 2015;348:56-61.

26 Deng L, Liang H, Burnette B, et al. Irradiation and anti-PD-L1 treatment synergistically promote antitumor immunity in mice. J Clin Invest 2014;124:687-95.

27 Wan C, Sun Y, Tian Y, et al. Irradiated tumor cell-derived microparticles mediate tumor eradication via cell killing and immune reprogramming. Sci Adv 2020;6:eaay9789.

28 Wang L, Rubinstein R, Lines JL, et al. VISTA, a novel mouse Ig superfamily ligand that negatively regulates $T$ cell responses. $J$ Exp Med 2011;208:577-92.

29 Gao J, Ward JF, Pettaway CA, et al. VISTA is an inhibitory immune checkpoint that is increased after ipilimumab therapy in patients with prostate cancer. Nat Med 2017;23:551-5.

30 Nowak EC, Lines JL, Varn FS, et al. Immunoregulatory functions of VISTA. Immunol Rev 2017;276:66-79.

31 Zong L, Zhang M, Wang W, et al. PD-L1, B7-H3 and VISTA are highly expressed in gestational trophoblastic neoplasia. Histopathology 2019;75:421-30.

32 Mulati K, Hamanishi J, Matsumura N, et al. VISTA expressed in tumour cells regulates T cell function. Br J Cancer 2019;120:115-27.

33 Altan M, Pelekanou V, Schalper KA, et al. B7-H3 expression in NSCLC and its association with B7-H4, PD-L1 and tumor-infiltrating lymphocytes. Clin Cancer Res 2017;23:5202-9.

34 Loeser H, Kraemer M, Gebauer F, et al. The expression of the immune checkpoint regulator VISTA correlates with improved overall survival in pT1/2 tumor stages in esophageal adenocarcinoma. Oncoimmunology 2019;8:e1581546.

35 Coy S, Rashid R, Lin J-R, et al. Multiplexed immunofluorescence reveals potential Pd-1/Pd-L1 pathway vulnerabilities in craniopharyngioma. Neuro Oncol 2018;20:i39-39.

36 Himes BT, Ruff MW, Van Gompel JJ, et al. Recurrent papillary craniopharyngioma with BRAF V600E mutation treated with dabrafenib: case report. J Neurosurg 2019;130:1299-303.

37 Liu R, Zhang T, Zhu G, et al. Regulation of mutant TERT by BRAF V600E/MAP kinase pathway through FOS/GABP in human cancer. Nat Commun 2018;9:1-13.

38 Deng J, Li J, Sarde A, et al. Hypoxia-Induced vista promotes the suppressive function of myeloid-derived suppressor cells in the tumor microenvironment. Cancer Immunol Res 2019;7:1079-90. 